\title{
The role of albumin-to-globulin ratio in undifferentiated arthritis: Rheumatoid arthritis versus primary Sjögren syndrome
}

\author{
Reyhan Köse Çobanoglu ${ }^{1}$, Taşkın Şentürk² \\ ${ }^{1}$ Rheumatology Clinic, Aydın State Hospital, Aydın, Türkiye \\ ${ }^{2}$ Department of Internal Medicine, Division of Rheumatology, Adnan Menderes University Faculty of Medicine, Aydin, Türkiye
}

\begin{abstract}
Objectives: This study aims to compare initial albumin-to-globulin ratio (AGR) in patients with rheumatoid arthritis (RA) and primary Sjögren syndrome (pSS) presenting with undifferentiated arthritis (UA) and to investigate whether there was a difference in terms of AGR between the two patient groups and healthy controls.

Patients and methods: Between January 2019 and December 2019, a total of 177 patients including 96 RA (10 males, 86 females; mean age: $53.6 \pm 10.8$ years; range, 21 to 74 years) and 81 pSS (5 males, 76 females; mean age: $53.2 \pm 14.1$ years; range, 23 to 79 years) and 82 healthy controls ( 20 males, 62 females; mean age: $50.5 \pm 13.6$ years; range, 20 to 79 years) were included in this case-control study. Demographic characteristics, albumin, and globulin levels of all participants were recorded. The AGR, erythrocyte sedimentation rate (ESR), C-reactive protein (CRP), rheumatoid factor (RF), anti-nuclear antibody (ANA), and anti-citrullinated protein antibodies (ACPA) were assessed.

Results: The mean AGR was $1.50 \pm 0.16$ in the control group, $1.48 \pm 0.24$ in the RA group, and $1.30 \pm 0.23$ in the pSS group, indicating a significant difference between the pSS and the other two groups $(p<0.001)$. The receiver operating characteristic analysis revealed that the cut-off value for $A G R$ was 1.39 (area under the curve $=0.736$ ) with a sensitivity of 0.642 and a specificity of $0.646(p<0.001)$. The ESR and CRP values were higher $(p<0.001)$, and ANA $(p<0.001)$ and RF $(p=0.003)$ positivity were lower in the RA group, compared to the pSS group.

Conclusion: This study findings indicate that AGR is a helpful tool in the differential diagnosis of RA and pSS presenting with UA at the time of admission, and Sjögren syndrome should be considered in case of $A G R \leq 1.39$.

Keywords: Albumin-to-globulin ratio, primary Sjögren syndrome, rheumatoid arthritis, undifferentiated arthritis.
\end{abstract}

Undifferentiated arthritis (UA) is an umbrella term of early inflammatory arthritis that does not meet the diagnostic or classification criteria of any certain disease. Patients presenting with UA may be suffered from mono/oligo- or polyarticular arthritis and are usually in the first several weeks to months of symptom duration. Undifferentiated arthritis may have different outcomes such as a definite rheumatic disease, a self-limited arthritis or remains as UA. Most of the polyarticular involvements progressed to rheumatoid arthritis
(RA)., ${ }^{1,2}$ In other forms of polyarthritis such as systemic lupus erythematosus, dermatomyositis/ polymyositis, systemic sclerosis and psoriatic arthritis, extraarticular findings are prevalent and many clues to the diagnosis are seen in the early period. ${ }^{3}$

Rheumatoid arthritis and primary Sjögren syndrome (pSS), which are common connective tissue diseases, may share many clinical features. The latter is a chronic systemic autoimmune disorder that usually affects women aged

Received: January 19, 2021 Accepted: August 23, 2021 Published online: December 24, 2021

Correspondence: Reyhan Köse Çobanoglu, MD. Aydın Devlet Hastanesi, Romatoloji Kliniği, 09100 Efeler, Aydın, Türkiye. Tel: +90 533 - 5187031 e-mail: reyhan_kose@yahoo.com

\section{Citation:}

Köse Çobanoglu R, Şentürk T. The role of albumin-to-globulin ratio in undifferentiated arthritis: Rheumatoid arthritis versus primary Sjögren syndrome. Arch Rheumatol 2022;37(2):245-251. 
40 to 60 years. Lymphocytic infiltration of salivary, lacrimal, and other exocrine glands is typical and extraglandular involvement may also occur. Before clinical pSS is clearly evident, early signs are non-specific and usually include arthralgia-arthritis, fatigue, and dry eyes and/or mouth. ${ }^{4}$ In pSS, articular manifestations usually present as symmetric polysynovitis mimicking RA; unfortunately, the presence of erosion is the only definitive differential diagnostic sign. ${ }^{5}$ Since these two entities have different courses and outcomes, differential diagnosis in the early period is crucial.

Albumin is synthesized in the liver and constitutes almost more than half of serum proteins. It provides $70 \%$ of the intravascular colloid osmotic pressure and acts as a transporter for many hormones, bilirubin, and medications. Moreover, it reflects nutritional status and is a negative acute phase reactant. ${ }^{6}$ Globulins include numerous serum proteins such as enzymes, complementary proteins and immunoglobulins, and have an important role in the inflammation cascade, indicating the level of circulating immunoglobulins. ${ }^{6}$ There are many conditions such as dehydration, inflammation, and malnutrition that affect albumin and globulin levels. The albumin-to-globulin ratio (AGR), calculated as AGR=albumin/globulin, has been extensively studied for cancer survival and prognosis, and also studied in cardiovascular diseases. Lower AGR has been found to be significantly associated with poor outcomes. ${ }^{7,8}$ The AGR has not been a well-studied variable in rheumatic diseases. In an earlier study, it was found to be a predictor of the clinical stages of sarcoidosis. ${ }^{9}$

In the present study, we aimed to compare initial AGR in the patients with RA and pSS presenting with UA and to investigate whether there was a difference in terms of AGR between the two patient groups.

\section{PATIENTS AND METHODS}

This single center, case-control study was conducted at Adnan Menderes University Faculty of Medicine, Department of Rheumatology between January 2019 and December 2019. The patients with early/UA who fulfilled the RA or pSS classification criteria of American College of Rheumatology/European League Against Rheumatism (ACR/EULAR) criteria and followed for two years were included. The control group consisted of age-matched healthy individuals. The diagnosis of RA was made based on the 2010 ACR classification criteria, ${ }^{10}$ while the diagnosis of pSS was made based on the 2016 ACR/EULAR criteria. ${ }^{11}$ Patients under the age of 20 or over 79 years, patients with a history of infection or malignancy, kidney and liver failure, uncontrolled metabolic disease, proven chronic inflammatory disease previously, and patients using steroid at a dose of $>10 \mathrm{mg} /$ day within the past four weeks were excluded from the study. Finally, a total of 177 patients including 96 RA (10 males, 86 females; mean age: $53.6 \pm 10.8$ years; range, 21 to 74 years) and 81 pSS (5 males, 76 females; mean age: $53.2 \pm 14.1$ years; range, 20 to 79 years) and 82 healthy controls $(20$ males, 62 females; mean age: $50.5 \pm 13.6$ years; range, 23 to 79 years) were included in the study.

The differential diagnosis was made by evaluating the erosive changes of the disease on direct radiographs during follow-up. All participants' laboratory tests were assessed in venous blood samples, and blood samples were drawn between 9:00 AM and 11:00 AM following an 8-h overnight fasting. Total protein and albumin were measured spectrophotometrically (Abbott Architect ${ }^{\circledR}$ Plus ci16200; Abbott Park, IL, USA). Serum globulin was measured as the difference between serum total protein and serum albumin. Data including demographic characteristics, physical examination, history of drug use, total protein, albumin, and globulin levels of both the patients and healthy controls were obtained from medical records. Erythrocyte sedimentation rate (ESR), C-reactive protein (CRP), symptom duration, and number and pattern of joints affected at the time of presentation ${ }^{12}$ were assessed. Rheumatoid factor (RF), anti-nuclear antibody (ANA), and anti-citrullinated protein antibodies (ACPA) of the RA and pSS patients were noted. The AGR was calculated as serum albumin/ (total protein-albumin).

\section{Statistical analysis}

Statistical analysis was performed
using the SPSS version 17.0 for Windows


(SPSS Inc., Chicago, IL, USA). Descriptive data were expressed in mean \pm standard deviation (SD), median (min-max) or number and frequency. The Kolmogorov-Smirnov test was used to assess the normality of numeric variables. For the numerical variables that were normally distributed, comparison among groups was performed with analysis of variance (ANOVA). For the numerical variables that were not normally distributed, comparison between the groups was performed using the KruskalWallis test and the Mann-Whitney $U$ test. In case of a difference between the groups, the Bonferroni post-hoc test was applied to determine which group caused the difference. Comparison of qualitative data was performed using the chi-square test. The receiver operating characteristic (ROC) curves were plotted to estimate the optimal cut-off value, as well as the sensitivity, specificity, positive predictive value (PPV), and negative predictive value (NPV) for AGR. The correlation between the ESR, CRP, age and AGR was analyzed using the Spearman correlation (rho) analysis. Accordingly, a value of rho 0.0 to 0.19 was accepted as very weak, 0.2 to 0.39 as weak, 0.4 to 0.59 as moderate, 0.6 to 0.79 as strong, and 0.80 to 1.0 as very strong. ${ }^{13}$ A $p$ value of $<0.05$ was considered statistically significant.

\section{RESULTS}

A total of 177 patients with RA or pSS and 82 healthy controls were included in the study. There was no statistically significant difference among the three groups in terms of age $(p=0.233)$. However, the number of male patients was statistically significantly higher in the control group ( $p=0.001$ ), while no significant difference was observed between the RA and pSS groups $(p=0.231)$ (Table 1).

The inter-group analysis for albumin showed a significant difference between the control and the other two groups $(p=0.001)$; however, no significant difference was found between the RA and pSS groups. The inter-group analysis for globulin showed a significant difference between the pSS and the other two groups $(p<0.001)$, while there was no significant difference between the control and RA groups. The mean AGR was $1.50 \pm 0.16$ in healthy controls, $1.48 \pm 0.24$ in RA group, and $1.30 \pm 0.23$ in pSS group, indicating a statistically significant difference among the groups $(p<0.001)$. In the post-hoc analysis, pSS was significantly different from the control group $(p<0.001)$ and RA group $(p<0.001)$ (Table 2).

Table 1. Demographic and clinical characteristics of RA and pSS patients

\begin{tabular}{|c|c|c|c|c|c|c|c|c|c|c|c|}
\hline & \multicolumn{5}{|c|}{ RA patients $(n=96)$} & \multicolumn{5}{|c|}{ pSS patients $(\mathrm{n}=81)$} & \multirow[b]{2}{*}{$p^{*}$} \\
\hline & $\mathrm{n}$ & $\%$ & Mean \pm SD & Median & Min-Max & $\mathrm{n}$ & $\%$ & Mean \pm SD & Median & Min-Max & \\
\hline Age (year) & & & $53.6 \pm 10.8$ & & & & & $53.2 \pm 14.1$ & & & 0.978 \\
\hline $\begin{array}{l}\text { Sex } \\
\quad \text { Male } \\
\text { Female }\end{array}$ & $\begin{array}{l}10 \\
86\end{array}$ & $\begin{array}{l}10.5 \\
89.5\end{array}$ & & & & $\begin{array}{c}5 \\
76\end{array}$ & $\begin{array}{c}6.2 \\
93.8\end{array}$ & & & & 0.231 \\
\hline Symptom duration (week) & & & & 14 & $3-46$ & & & & 17 & $3-43$ & 0.326 \\
\hline $\begin{array}{l}\text { Number of joints } \\
\quad 1-3 \\
4-10 \\
>10\end{array}$ & $\begin{array}{c}70 \\
20 \\
6\end{array}$ & $\begin{array}{c}72.9 \\
20.8 \\
6.3\end{array}$ & & & & $\begin{array}{c}63 \\
14 \\
4\end{array}$ & $\begin{array}{c}77.8 \\
17.3 \\
4.9\end{array}$ & & & & 0.474 \\
\hline $\begin{array}{l}\text { Pattern of joints } \\
\text { Upper symmetric } \\
\text { Lower symmetric } \\
\text { Upper asymmetric } \\
\text { Lower asymmetric } \\
\text { Upper and low }\end{array}$ & $\begin{array}{c}54 \\
6 \\
29 \\
2 \\
5\end{array}$ & $\begin{array}{l}56.25 \\
6.25 \\
30.2 \\
2.1 \\
5.2\end{array}$ & & & & $\begin{array}{c}45 \\
9 \\
22 \\
1 \\
4\end{array}$ & $\begin{array}{c}55.6 \\
11.1 \\
27.2 \\
1.2 \\
4.9\end{array}$ & & & & 0.783 \\
\hline
\end{tabular}


Table 2. Inter-group comparison of baseline albumin, globulin, and AGR

\begin{tabular}{|c|c|c|c|c|c|c|c|c|}
\hline & \multirow[b]{2}{*}{$\mathrm{n}$} & \multicolumn{2}{|c|}{ RA patients $(n=96)$} & \multicolumn{2}{|c|}{ pSS patients $(\mathrm{n}=81)$} & \multicolumn{2}{|c|}{ Healthy controls $(n=82)$} & \multirow[b]{2}{*}{$p^{*}$} \\
\hline & & Median & Min-Max & Median & Min-Max & Median & Min-Max & \\
\hline Albumin & 259 & 4.1 & $3.5-4.8$ & 4.2 & $3.4-4.8$ & $4.3 b$ & $3.7-4.9 \dagger$ & 0.001 \\
\hline Globulin & 259 & 2.84 & 0.45 & $3.27 \neq$ & 0.52 & 2.8 & 2.3-3.6 & $<0.001$ \\
\hline AGR & 259 & 1.48 & 0.24 & $1.3^{\mathrm{T}}$ & 0.23 & 1.5 & 0.16 & $<0.001$ \\
\hline
\end{tabular}

RA: Rheumatoid arthritis; pSS: Primary Sjögren syndrome; AGR: Albumin-to-globulin ratio; * One-way analysis of variance and Kruskal-Wallis test; † Control group was significantly different from the pSS $(p=0.026)$ and RA ( $p=0.001)$ groups; $\neq \mathrm{pSS}$ group was significantly different from the control $(p<0.001)$ and RA ( $<<0.001)$ groups; TI pSS group was significantly different from the control $(p<0.001)$ and RA $(p<0.001)$ groups.

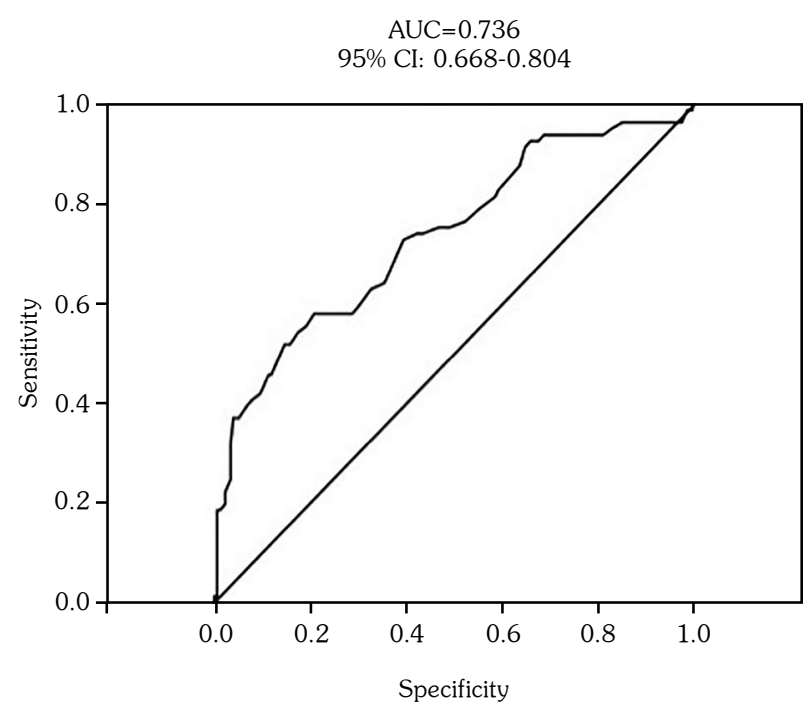

Figure 1. Receiver operating characteristic analysis of albumin-to-globulin ratio for undifferentiated arthritis progressed to primary Sjögren syndrome. Area under curve (AUC) and 95\% confidence interval (CI) are indicated.
In the ROC analysis, the area under curve (AUC) of AGR for UA progressed to pSS was 0.736 with a confidence interval $(\mathrm{CI})$ of 0.668 0.804 , and the cut-off value was $1.39(\mathrm{p}<0.001)$ with a sensitivity of $64.2 \%$, specificity of $64.6 \%$, PPV of $55 \%$, and NPV of $80 \%$ (Figure 1).

The mean symptom duration, and the number and pattern of joints involved were similar in RA and pSS patients (Table 1). However, the median ESR and CRP values were significantly higher in RA patients compared to the pSS group $(p<0.001)$. Autoantibody positivity ratio was found to be different between the two groups, except for ACPA. The RF positivity was $8.3 \%$ and $24.7 \%$ in $\mathrm{RA}$ and $\mathrm{pSS}$ groups, respectively $(\mathrm{p}=0.03)$. The ANA positivity was $20.8 \%$ and $63 \%$ in RA and pSS groups, respectively $(p<0.001)$. The ACPA positivity was similar and all was in the low titers in both groups. Serological parameters of RA and pSS patients are shown in Table 3.

Table 3. Serological parameters between RA and pSS groups

\begin{tabular}{|c|c|c|c|c|c|c|c|c|c|}
\hline & \multicolumn{4}{|c|}{ RA patients $(n=96)$} & \multicolumn{4}{|c|}{ pSS patients $(\mathrm{n}=81)$} & \multirow[b]{2}{*}{$p^{*}$} \\
\hline & $\mathrm{n}$ & $\%$ & Median & Min-Max & $\mathrm{n}$ & $\%$ & Median & Min-Max & \\
\hline $\mathrm{ESR}(\mathrm{mm} / \mathrm{h})$ & & & 38 & $4-87$ & & & 24 & $3-91$ & $<0.001$ \\
\hline CRP (mg/dL) & & & 9.2 & $0.4-63.6$ & & & 2 & $0.6-62$ & $<0.001$ \\
\hline RF positivity & 8 & 8.3 & & & 20 & 24.7 & & & 0.003 \\
\hline ACPA positivity & 6 & 6.3 & & & 5 & 6.2 & & & 0.617 \\
\hline ANA positivity & 20 & 20.8 & & & 51 & 63 & & & $<0.001$ \\
\hline
\end{tabular}

RA: Rheumatoid arthritis; pSS: Primary Sjögren syndrome; ESR: Erythrocyte sedimentation rate; CRP: C-reactive protein; RF: Rheumatoid factor; ACPA: Anti-citrullinated protein antibody; ANA: Anti-nuclear antibody; * Chi-square test and Mann-Whitney U test. 
According to the correlation analysis, the AGR was negatively correlated with the ESR (rho=-0.15, p=0.04), but positively correlated with ESR and CRP (rho=0.42, $\mathrm{p}<0.001)$. There was no significant correlation between the age and AGR, CRP, AGR.

\section{DISCUSSION}

In the rheumatology practice, it may be a challenge to differentiate the diagnosis of the patients, particularly of those presenting with similar symptoms originating from different diseases. Biomarkers play an important role in diagnosis and determining the prognosis of diseases; however, there is no specific marker available for any rheumatic disease. In the present study, we found a significant difference between the two patient groups in terms of baseline AGR. Pre-treatment AGR is widely used to evaluate cancer survival rate and prognosis. ${ }^{7}$ A meta-analysis evaluating 28 studies involving 15,356 cancer patients found that cancer patients with low pre-treatment AGR had a decreased survival rate and an increased rate of cancer recurrence or progression. ${ }^{7}$ Furthermore, AGR was evaluated in heart failure with reduced ejection fraction in terms of prognosis, and lower AGR was associated with 90-day and one-year mortality rates. ${ }^{8}$

Albumin and globulin levels can be affected from many diseases and conditions such as liver diseases, infections, and dehydration. ${ }^{6}$ Changes in AGR in oncological patients have usually been suggested to associate with host-related factors such as malnutrition and cachexia, which decrease albumin levels, and with hematological malignancies that can increase globulin. ${ }^{8}$ Albumin is a negative acute phase reactant, and fluid redistribution that affects plasma albumin levels has been proposed to be partly implicated in the underlying mechanism of changes in AGR in heart failure..$^{7}$ On the other hand, AGR has not been well studied in rheumatic diseases so far, and there is only one study investigating AGR in rheumatic diseases in the literature. Nandedkar et al. ${ }^{9}$ found AGR to be correlated with the clinical stages of sarcoidosis. They divided patients according to their clinical stages as Stage I, Stage II, and Stage III. The AGR varied between the stages with a significant difference, and lower AGR was correlated with the advanced clinical stages of sarcoidosis. In our study, lower AGR was found to be a predictor of UA progressing to $\mathrm{pSS}$.

Although RA and pSS may emerge with a similar clinical presentation, there are certain differences in the immunogenetic pathogenesis. Chronic inflammatory processes in autoimmune diseases, including RA and pSS, may cause polyclonal increase in immunoglobulin levels. Nevertheless, pSS presents a fundamental prototype of autoimmune diseases in which CD4+ $\mathrm{T}$ and $\mathrm{B}$ lymphocytes play the main role. ${ }^{14,15}$ Production of autoantibodies in pSS is the hallmark of the disease. Autoantibodies against Ro/SSA and La/SSB antigens, which are most commonly identified in pSS, are rarely detected in patients with RA and in patients with SS secondary to RA. ${ }^{4,14,15}$ Therefore, hypergammaglobulinemia is a rather common finding in $\mathrm{pSS} .{ }^{16}$ In the light of these data, we believe that lower AGR in UA patients who progress to $\mathrm{pSS}$ is related to these pathogenetic mechanisms.

Rheumatoid factor may be present in many autoimmune conditions, lymphoproliferative diseases, and infections. Additionally, RF positivity has a similar frequency in both RA and pSS and can be detected up to 60 to $70 \%$ of cases. ${ }^{17}$ In our study, patients with UA who progressed to RA mostly remained seronegative and, thus, the pSS patients more frequently exhibited RF positivity. In a study showing that most UA patients were finally classified as RA, the RF and/or ACPA positivity in UA patients was found to be $10 \%{ }^{12}$ It is well known that ACPA is highly specific for $\mathrm{RA}$; in contrast, $\mathrm{ACPA}$ positivity rate in $\mathrm{pSS}$ is only 5 to $10 \%{ }^{18,19}$ that is considered to be a risk factor for the development of synovitis in pSS and progression to RA. ${ }^{5,20}$ In our study, the ACPA positivity rate was similar in both groups: all were at low titers and no pSS patient with ACPA developed RA.

In our study, joint involvements were mostly in the upper extremities and were usually symmetrical. In an early arthritis cohort with 776 patients, $76 \%$ of patients had upper extremity involvement, mostly in the hands, and $48 \%$ had symmetrical joint involvement, ${ }^{12}$ consistent with our study. Acute phase reactants were also 
found to be significantly higher in RA patients at baseline. In a study investigating the risk factors of developing RA in patients with ACPA-positive $\mathrm{pSS}$, the only variable was the elevation of the baseline acute phase reactants. ${ }^{5}$ The authors considered that patients initially diagnosed with pSS might have RA-associated SS. In some studies comparing the clinical characteristics of seronegative and seropositive RA patients, those with seronegative disease were found to have a higher disease activity at baseline. ${ }^{21,22}$ These results were attributed to the fact that the number of joints involved in seronegative disease were higher compared to seropositive disease, which requires to fulfill the 2010 classification criteria of RA. In our study, it was unclear why there was a difference in the baseline acute phase values between RA and pSS patients presenting with UA; however, it can be due to synovitis in other joints that are not clinically overt or to subclinical erosive disease.

An increase in plasma proteins, such as fibrinogen and globulins, causes elevated ESR. ${ }^{23}$ A decline in AGR is expected in patients having elevated globulin levels. Consistently, AGR was negatively correlated with ESR in our study.

There are some limitations to this study. Given the retrospective nature of the study, clinical outcomes of all our UA patients were unable to be evaluated. As the main clinical considerations in this study were connective tissue diseases, we only included RA and pSS that cause the most confusion and can be misdiagnosed in clinical practice. In addition, we were unable to evaluate all patients in the early period with imaging methods such as ultrasonography or magnetic resonance imaging and, thus, a UA patient with an uncertain joint involvement might have been classified as RA or another disease.

In conclusion, patients presenting with similar symptoms, particularly who have seronegative disease or low-titer antibody positivity, AGR may be a helpful tool in the differential diagnosis of RA and pSS. An AGR of $\leq 1.39$ may be suggestive of the presence of SS and, therefore, this may guide the management of the diseases in the early period. We believe that AGR may be also used as a prognostic and predictive marker in rheumatic diseases. As in UA progression, it also may be useful in certain rheumatic disease for predicting the course of the disease, whether there would be systemic involvement. Nevertheless, further largescale, prospective, randomized-clinical studies are needed to draw reliable conclusions on this subject.

Ethics Committee Approval: The study protocol was approved by the Adnan Menderes University Faculty of Medicine Ethics Committee (No: 2020/25). The study was conducted in accordance with the principles of the Declaration of Helsinki.

Data Sharing Statement: The data that support the findings of this study are available from the corresponding author upon reasonable request.

Author Contributions: Conception, writing design, material, data collection: R.K.Ç.; critical review, supervision: T.Ş.

Conflict of Interest: The authors declared no conflicts of interest with respect to the authorship and/or publication of this article.

Funding: The authors received no financial support for the research and/or authorship of this article.

\section{REFERENCES}

1. Verpoort KN, van Dongen H, Allaart CF, Toes RE, Breedveld FC, Huizinga TW. Undifferentiated arthritis-disease course assessed in several inception cohorts. Clin Exp Rheumatol 2004;22(5 Suppl 35):S12-7.

2. Rodríguez-Muguruza $\mathrm{S}$, Martínez-Morillo $\mathrm{M}$, Riveros-Frutos A, Tena X. What is the outcome of undifferentiated arthritis? Reumatol Clin 2015;11:57-8.

3. Dao K, Cush JJ. Acute polyarthritis. Best Pract Res Clin Rheumatol 2006;20:653-72.

4. Mavragani CP, Moutsopoulos HM. Sjögren syndrome. CMAJ 2014;186:E579-86.

5. Payet J, Belkhir R, Gottenberg JE, Bergé E, Desmoulins F, Meyer O, et al. ACPA-positive primary Sjögren's syndrome: True primary or rheumatoid arthritis-associated Sjögren's syndrome? RMD Open 2015;1:e000066.

6. Busher JT. Serum Albumin and Globulin. In: Walker HK, Hall WD, Hurst JW, editors. Clinical methods: The history, physical, and laboratory examinations. Chapter 101. 3rd ed. Boston: Butterworths; 1990. p. 497-9.

7. Lv GY, An L, Sun XD, Hu YL, Sun DW. Pretreatment albumin to globulin ratio can serve as a prognostic marker in human cancers: A meta-analysis. Clin Chim Acta 2018;476:81-91.

8. Niedziela JT, Hudzik B, Szygula-Jurkiewicz B, Nowak JU, Polonski L, Gasior M, et al. Albuminto-globulin ratio as an independent predictor of 
mortality in chronic heart failure. Biomark Med 2018;12:749-57.

9. Nandedkar AK, Royal GC Jr, Nandedkar MA. Evaluation of albumin-globulin ratio to confirm the clinical stages of sarcoidosis. J Natl Med Assoc 1986;78:969-71.

10. Aletaha D, Neogi T, Silman AJ, Funovits J, Felson DT, Bingham CO 3rd, et al. 2010 Rheumatoid arthritis classification criteria: An American College of Rheumatology/European League Against Rheumatism collaborative initiative. Arthritis Rheum 2010;62:2569-81.

11. Shiboski CH, Shiboski SC, Seror R, Criswell LA, Labetoulle M, Lietman TM, et al. 2016 American College of Rheumatology/European League Against Rheumatism classification criteria for primary Sjögren's syndrome: A consensus and data-driven methodology involving three international patient cohorts. Arthritis Rheumatol 2017;69:35-45.

12. Krabben A, Huizinga TW, van der Helm-van Mil $\mathrm{AH}$. Undifferentiated arthritis characteristics and outcomes when applying the 2010 and 1987 criteria for rheumatoid arthritis. Ann Rheum Dis 2012;71:238-41.

13. Akoglu H. User's guide to correlation coefficients. Turk J Emerg Med 2018;18:91-3.

14. Christodoulou MI, Kapsogeorgou EK, Moutsopoulos HM. Characteristics of the minor salivary gland infiltrates in Sjögren's syndrome. J Autoimmun 2010;34:400-7.

15. Nocturne G, Mariette X. B cells in the pathogenesis of primary Sjögren syndrome. Nat Rev Rheumatol 2018;14:133-45.

16. Brito-Zerón P, Perez-Alvarez R, Perez-de-Lis M, HidalgoTenorio C, Ramos-Casals M. Laboratory abnormalities in primary Sjögren's syndrome. In: Ramos-Casals M, Stone JH, Moutsopoulos HM, editors. Sjögren's syndrome. London: Springer; 2012. p. 347-66.
17. Tzioufas T, Youinou P, Moutsopoulos HM. Sjögren's syndrome. In: Maddison PJ, Isenberg DA, Woo P, Glass DN, editors. Oxford textbook of rheumatology. 2nd ed. Oxford: Oxford Medical Publications; 1997. p. 1301-17.

18. Vittecoq $\mathrm{O}$, Incaurgarat $\mathrm{B}$, Jouen-Beades F, Legoedec J, Letourneur O, Rolland D, et al. Autoantibodies recognizing citrullinated rat filaggrin in an ELISA using citrullinated and noncitrullinated recombinant proteins as antigens are highly diagnostic for rheumatoid arthritis. Clin Exp Immunol 2004;135:173-80.

19. Fabien N, Olsson NO, Goetz J, Johanet C, Escande A, Bardin N, et al. Prevalence of autoantibodies to cyclic citrullinated peptide in patients with rheumatic diseases other than rheumatoid arthritis: A French multicenter study. Clin Rev Allergy Immunol 2008;34:40-4.

20. Atzeni F, Sarzi-Puttini P, Lama N, Bonacci E, Bobbio-Pallavicini F, Montecucco C, et al. Anticyclic citrullinated peptide antibodies in primary Sjögren syndrome may be associated with non-erosive synovitis. Arthritis Res Ther 2008;10:R51.

21. Choi S, Lee KH. Clinical management of seronegative and seropositive rheumatoid arthritis: A comparative study. PLoS One 2018;13:e0195550.

22. Nordberg LB, Lillegraven S, Lie E, Aga AB, Olsen IC, Hammer $\mathrm{HB}$, et al. Patients with seronegative RA have more inflammatory activity compared with patients with seropositive RA in an inception cohort of DMARD-naïve patients classified according to the 2010 ACR/EULAR criteria. Ann Rheum Dis 2017;76:341-5.

23. Talstad I, Haugen HF. The relationship between the erythrocyte sedimentation rate (ESR) and plasma proteins in clinical materials and models. Scand J Clin Lab Invest 1979;39:519-24. 